\title{
Dopamine Controls the Neural Dynamics of Memory Signals and Retrieval Accuracy
}

\author{
Thore Apitz' and Nico Bunzeck*,' \\ 'Department of Systems Neuroscience, University Medical Center Hamburg-Eppendorf, Hamburg, Germany
}

\begin{abstract}
The human brain is capable of differentiating between new and already stored information rapidly to allow optimal behavior and decision-making. Although the neural mechanisms of novelty discrimination were often described as temporally constant (ie, with specific latencies), recent electrophysiological studies have demonstrated that the onset of neural novelty signals (ie, differences in event-related responses to new and old items) can be accelerated by reward motivation. While the precise physiological mechanisms underlying this acceleration remain unclear, the involvement of the neurotransmitter dopamine in both novelty and reward processing suggests that enhanced dopamine levels in the context of reward prospect may have a role. To investigate this hypothesis, we used magnetoencephalography (MEG) in combination with an old/new recognition memory task in which correct discrimination between old and new items was rewarded. Importantly, before the task, human subjects received either $150 \mathrm{mg}$ of the dopamine precursor levodopa or placebo. For the placebo group, old/new signals peaked at $\sim 100 \mathrm{~ms}$ after stimulus onset over left temporal/occipital sensors. In contrast, after levodopa administration earliest old/new effects only emerged after $\sim 400 \mathrm{~ms}$ and retrieval accuracy was reduced as expressed in lower $d^{\prime}$ values. As such, our results point towards a previously unreported role of dopamine in controlling the chronometry of neural processes underlying the distinction between old and new information. They also suggest that this relationship follows a nonlinear function whereby slightly enhanced dopamine levels accelerate neural/cognitive processes and excessive dopamine levels impair them.

Neuropsychopharmacology (2013) 38, 2409-24I7; doi:I0.1038/npp.2013.14I; published online 26 June 2013
\end{abstract}

Keywords: reward; motivation; novelty; magnetoencephalography; dopamine

\section{INTRODUCTION}

The ability to distinguish rapidly between old and new information is critical for adaptive behavior and requires suitable neural mechanisms. Indeed, during recognition memory, the human brain distinguishes between old (ie, repeated) and new items already at $\sim 200 \mathrm{~ms}$ after stimulus onset (for a review see Rugg and Curran, 2007). Despite these consistently reported onset latencies, it is also known that scalp-recorded old/new responses can be accelerated from $\sim 200$ to $\sim 85 \mathrm{~ms}$ after stimulus onset if correct old/new decisions are monetarily rewarded (Bunzeck et al, 2009). These findings show that the chronometry of neural novelty processing (ie, differences in event-related responses to old $v s$ new items) can be modulated by reward motivation, and they suggest that such acceleration might be due to a functional interaction between novelty and reward, which is based on common neural substrates in the dopaminergic mesolimbic system. Support for this notion

\footnotetext{
*Correspondence: Dr N Bunzeck, Department of Systems Neuroscience, University Medical Center Hamburg-Eppendorf, Martinistrasse 52, Hamburg 20246, Germany, Tel: +49 04074105 9902, Fax: +4904074l05 9955, E-mail: n.bunzeck@uke.de Received 20 March 2013; revised 27 May 2013; accepted 28 May 20 13; accepted article preview online 3 June 2013
}

comes from a recent study using magnetoencephalography (MEG) together with a non-rewarded memory task (Eckart and Bunzeck, 2013). It revealed that stimulating the dopaminergic system through levodopa leads to faster novelty responses during the incidental encoding of repeatedly presented scene images.

Dopamine neurons in the substantia nigra/ventral tegmental area (SN/VTA) and interconnected medial temporal lobe (MTL) signal both novelty and reward. For instance, the human SN/VTA is activated by monetary incentives and novel images, as well as by cues that predict their occurrence (Bunzeck et al, 2013; O'Doherty, 2004; Wittmann et al, 2007). Furthermore, the MTL, which has long been known to be critical for novelty detection (Brown and Xiang, 1998; Strange et al, 1999; Yamaguchi et al, 2004), is also implicated in various forms of reward processing (Devenport et al, 1981). For example, the rodent hippocampus shows increased activity in baited maze arms (Holscher et al, 2003); in non-human primates, it is involved in learning place-reward associations (Rolls and Xiang, 2005), and in humans, it represents value during learning (Wimmer and Shohamy, 2012).

Here, we combined psychopharmacology with a previously established recognition memory paradigm (Bunzeck et al, 2009) to test the role of dopamine in controlling the temporal dynamics of neural novelty 
discrimination. Human subjects either received the dopamine precursor levodopa or placebo, and subsequently performed a reward-based old/new discrimination task while their brain activity was scanned using MEG. In the placebo group, we expected reward-driven early old/new effects (peaking at $\sim 100 \mathrm{~ms}$ ) over temporal/occipital sensors (Bunzeck et al, 2009) that would be modulated by levodopa. One hypothesis was that stimulating the dopaminergic system leads to further acceleration indicating a monotonic relationship between dopamine levels and the onset of neural novelty responses (Eckart and Bunzeck, 2013).

Alternatively, the relationship between dopamine levels and onset latencies could follow an inverted u-shaped function as described for other cognitive domains, such as working memory (Cools and D'Esposito, 2011; GoldmanRakic et al, 2000). Therefore, in the context of reward prospect, which is known be associated with dopamine release (Schott et al, 2008; Schultz et al, 1997), further stimulating the dopaminergic system might delay rather than accelerate the onset of neural novelty signals. Behaviorally, we expected corresponding changes in retrieval accuracy or reaction times (RTs).

\section{MATERIALS AND METHODS}

\section{Participants}

Thirty-eight human subjects participated in the experiment. All were randomly assigned to one of two experimental groups in a double-blind manner. Twenty participants (10 males and 10 females; age range $=21-34$ years; mean age $=26.4$ years; $\mathrm{SD}=3.53$ years) orally received the dopamine precursor levodopa ( $150 \mathrm{mg}$ levodopa, $37.5 \mathrm{mg}$ benserazid) before the task ('levodopa group'), while the other 18 participants ( 8 females and 10 males; age range $=18-33$ years; mean age $=24.6$ years; $\mathrm{SD}=4.19$ years) received a placebo ('placebo group'). Levodopa is licensed for the treatment of Parkinson's disease and provokes only little to no side effects if taken in low dosages. It has been used in previous imaging studies (eg, Guitart-Masip et al, 2012; Bunzeck et al, 2013; Eckart and Bunzeck, 2013).

All participants were healthy, right-handed, and had normal or corrected-to-normal vision. None of the participants reported a history of neurological, psychiatric, or medical disorders or any current medical problems. Furthermore, before the experiment all subjects were tested for alcohol (via saliva test) and drug consumption (ie, amphetamines, benzodiazepines, cocaine, opiates, and marijuana, via urine test); the tests were negative in all cases. Experimental groups did not differ significantly in nicotine consumption (see Supplementary Materials and Methods for details). Each subject gave written informed consent according to the approval of the local ethics committee (medical association Hamburg).

\section{Experimental Design and Task}

As levodopa reaches peak blood plasma concentration about 45-60 min after intake (Nyholm et al, 2012), the main task started $1 \mathrm{~h}$ after drug intake. To assess possible side effects, participants filled in subjective rating scales on three time points (T1: before drug administration; T2: $45 \mathrm{~min}$ after drug administration; and T3: at the end of the MEG experiment, $\sim 2 \mathrm{~h}$ after drug administration). No differences in subjective ratings between treatment groups were detected (see Supplementary Materials and Methods for details).

All participants performed three sets of (1) a familiarization phase followed by (2) a recognition memory task inside the MEG scanner (Figure 1).

(1) Familiarization phase: At the beginning of each experimental set, participants were familiarized with 20 indoor and 20 outdoor images. These images were each presented two times in randomized order for $1.5 \mathrm{~s}$ with a jittered interstimulus interval (ISI) of $1500 \pm 250 \mathrm{~ms}$. Participants indicated the indoor/outdoor status of the images with a button press of either the index finger or middle finger of the right hand.

(2) Recognition memory task: After a brief pause of $20 \mathrm{~s}$ following the familiarization phase, a recognition memory-based preference judgment task was performed. This task was further subdivided into two blocks, each consisting of 20 images from the familiarization phase ('old images') and 20 images not previously presented ('new images'), with a pause of $20 \mathrm{~s}$ between blocks. Old and new images served as either positive $(\mathrm{CS}+)$ or negative $(\mathrm{CS}-)$ reinforcers. Participants were instructed to make a preference judgment to each image through a two-choice button press indicating 'I prefer' (press with index finger of right hand) or 'I do not prefer' (press with middle finger of right hand) depending on the contingency between image category (old $v s$ new) and reinforcement value $(\mathrm{CS}+v s \mathrm{CS}-)$. The contingency was randomized and indicated at the beginning of each block by either 'Old pictures will be rewarded if preferred' (old images served as CS + and new images as $\mathrm{CS}-$ ) or 'New pictures will be rewarded if preferred' (new images served as CS + and old images as CS - ). It should be noted that preference judgments could only be made following initial (mental) old/new discrimination.

At the beginning of the experiment, participants were informed that correct 'I prefer' responses following a CS + led to a win of $€ 1$, whereas (incorrect) 'I prefer' responses following a CS - led to a loss of $€ 0.30$. Both correct 'I do not prefer' responses following a CS - and (incorrect) 'I do not prefer' responses following a CS + led to neither win nor loss. Old or new images were presented in randomized order with each picture being shown for $1.5 \mathrm{~s}$ on a gray background. Subsequently, a white fixation cross was presented for $1500 \pm 250 \mathrm{~ms}$, serving as an ISI. Feedback about all earning was given at the end of the experiment but not on a trial-by-trial basis. Before the experiment, participants were informed that only $50 \%$ of their earnings would be paid (ie, a maximum of $€ 20$ ).

For each set unique images were used, resulting in 120 old and 120 new images being used altogether. All stimuli were gray-scaled and normalized to a mean 

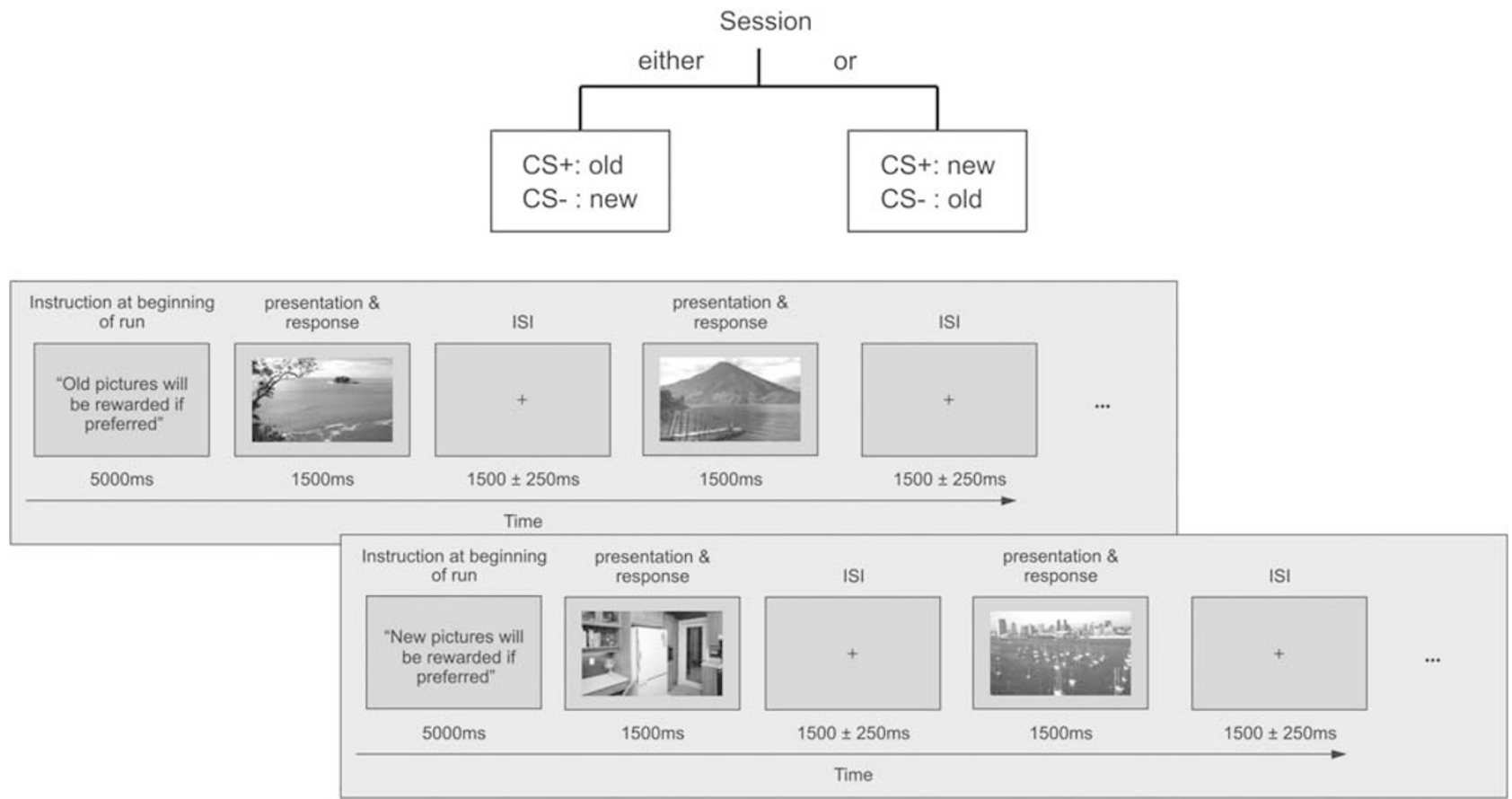

Figure I Experimental design. Subjects were first familiarized with a set of indoor and outdoor scene images (not shown) and then performed a rewardbased old/new discrimination task. See Materials and methods section for further details. CS, conditioned stimulus; ISI, interstimulus interval.

gray value of 127 and a standard deviation of 75 (8-bit grayscale, 0-255).

\section{Training Sessions}

Before the experiment, each participant completed two training sessions outside the MEG scanner. They were split into a familiarization phase and a recognition memory task including 10 old and 10 new images. In training session 1, a feedback was given on a trial-by-trial basis after each response (ie, $€ 1.00$; $€-0.30$; or $€ 0.00$ during the recognition memory task). Training session 2 resembled the main experiment, which means that reward feedback was not given immediately after each stimulus/response but at the end of the training session (ie, max. $€ 1.00$ ).

\section{Behavioral Data Analysis}

RTs during the recognition memory task were averaged across correct responses to old and new images for each trial of both the 'new rewarding/ old non-rewarding' and 'old rewarding/ new non-rewarding' contexts, resulting in RT scores for the conditions 'old and 'new'. These were entered into a $2 \times 2 \times 2$ analysis of variance (ANOVA) with the within-subject factors novelty (old images, new images), reward (reward predicting images, not-reward predicting images), and the between-subject factor group (levodopa, placebo).

Discrimination accuracy was assessed using the discrimination index $d^{\prime}$, which was calculated based on hit and false alarm rates (Stanislaw and Todorov, 1999). $D^{\prime}$ scores were analyzed using a $2 \times 2$ ANOVA with the betweensubject factor group (levodopa, placebo) and within-subject factor reward context (old rewarding/new non-rewarding, new rewarding/old non-rewarding).

\section{MEG Methods}

MEG recordings took place in a magnetically shielded room via a 275-channel CTF MEG system with SQUID-based axial gradiometers (VSM MedTech, Couquitlam, BC, Canada) and second-order gradients. Neuromagnetic signals were digitized continuously at a sampling rate of $1200 \mathrm{~Hz}$ and behavioral responses were made via an MEG-compatible response pad. Data were low-pass filtered at $240 \mathrm{~Hz}$ during acquisition and subsequently analyzed with SPM8 (Wellcome Trust Centre for Neuroimaging, University College, London, UK) and MATLAB software (The MathWorks, Natwick, MA, USA).

\section{Event-Related Responses}

MEG data were high-pass filtered at $0.25 \mathrm{~Hz}$ and low-pass filtered at $15 \mathrm{~Hz}$ using Butterworth filters. Subsequently, they were extracted from $200 \mathrm{~ms}$ before to $1000 \mathrm{~ms}$ after stimulus onset and baseline corrected relative to the $200 \mathrm{~ms}$ before stimulus onset (epoching). Epoched data were then downsampled at $150 \mathrm{~Hz}$, artifact detection was performed using simple thresholding to remove artifact-containing trials with signals exceeding $2500 \mathrm{fT}$ before averaging trials for each condition. Only trials with correct behavioral responses both during the familiarization and recognition memory task were used for averaging.

In a first step, we limited our analyses of the event-related magnetic fields (ERFs) to an a priori defined cluster of left occipital sensors, which previously revealed the strongest effects of reward on neural novelty processing (Bunzeck et al, 2009). These consisted of MLO24 and surrounding sensors (MLO12, MLO13, MLO14, MLO23, MLO33, MLO34, MLT27, MLT37, see Figure 2a). Regarding the time-window, 

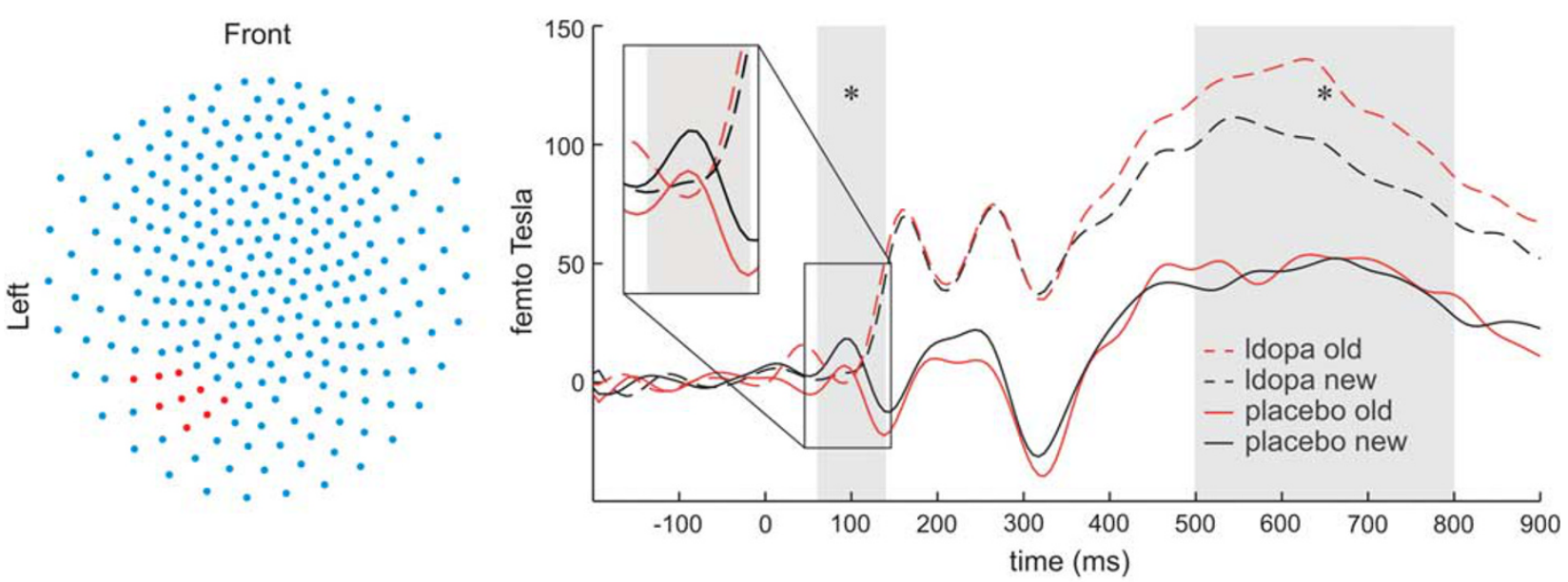

C

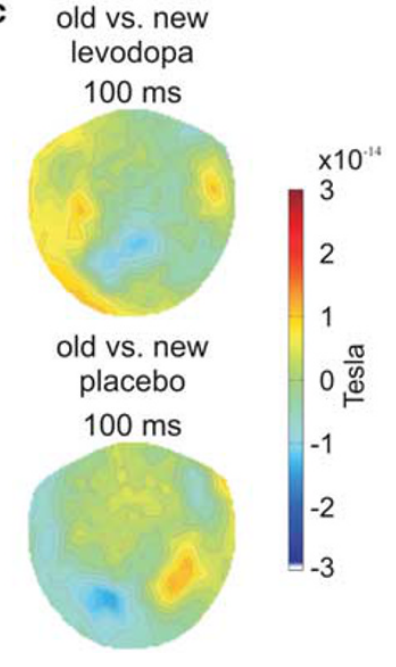

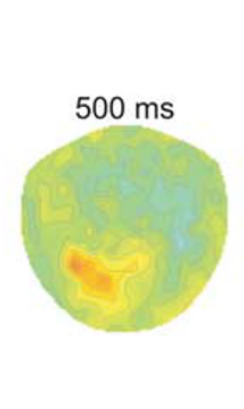

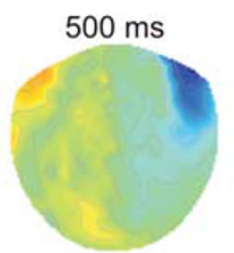

old vs. new

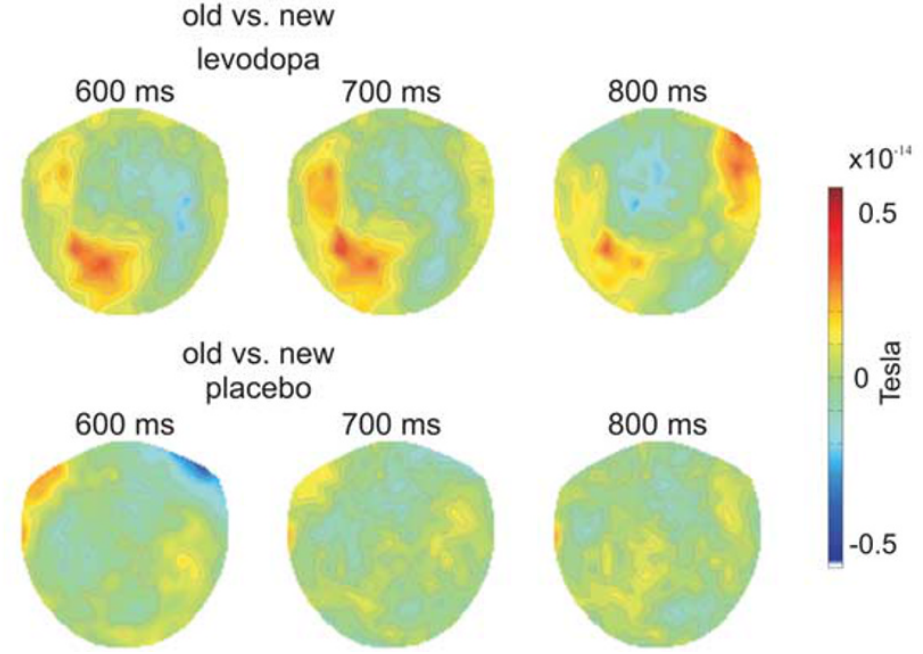

Figure 2 Interaction between novelty processing and drug group. Analyses of event-related magnetic fields were limited to a priori defined sensors of interest (red dots in (a), see text). Interactions between novelty status (old images, new images) and drug group (levodopa, placebo) were observed for an early $(60-140 \mathrm{~ms})$ and a later $(500-800 \mathrm{~ms})$ time-window (b). The early effect was driven by significant old/new responses in the placebo but not drug group; in the later time-window, the opposite pattern emerged. (c and d) Topographical maps of these effects (ie, differences between new vs old items). Asterisks represent statistically significant interactions $(p<0.05)$. For a full depiction of all eight conditions see Supplementary Figure SI.

we first focused on $60-140$ ms (Bunzeck et al, 2009) and, after visual inspection (see results), on $500-800 \mathrm{~ms}$. This later time-window is compatible with previous studies on old/new recognition memory (eg, Rugg and Curran, 2007). For both time-windows (60-140 and 500-800 ms), averaged ERFs for each condition and participant were entered into a $2 \times 2 \times 2$ ANOVA with the within-subject factors novelty (new images, old images), reward (reward predicting images, not-reward predicting images), and the betweensubject factor group (levodopa, placebo).

In a second, less hypothesis-driven analysis, we studied the effects of levodopa across all sensors and time-points (ie, the whole time-window ranging from -200 to $1000 \mathrm{~ms}$ after stimulus onset). Here, epoched and averaged data were converted into Neuroimaging Informatics Technology Initiative (NIfTI) format, producing one 3D image of channel space $\times$ time for each condition and participant. The 2D channel space was created by projecting sensor locations onto a plane followed by a linear interpolation to a
$64 \times 64$ pixel grid (pixel size $2.12 \times 2.69 \mathrm{~mm}^{2}$ ). The time dimension consisted of 181 samples per epoch with a length of $6.67 \mathrm{~ms}$ each. These images were smoothed using a Gaussian kernel (full-width half-maximum, FWHM) of FWHM $=5 \times 5 \times 15 \mathrm{~mm}^{3}$. Smoothing was carried out to allow for accommodating the spatial and/or temporal variance between participants; it also leads to a better conformity regarding random field theory (Worsley et al, 2004).

Subsequently, the 3D images for each condition and participant were entered into a $2 \times 2 \times 2$ ANOVA with the within-subject factors novelty (new images, old images) and reward (reward predicting images, not-reward predicting images) and the between-subject factor group (levodopa, placebo). This approach allowed us to test for main effects and interactions across all sensors and time-points including baseline. All second-level analyses in SPM8 were corrected for multiple comparisons using family-wise error correction. Further details on SPM8 for M/EEG can be found in Litvak et al (2011). 


\section{RESULTS}

All analyses (behavioral and MEG) are based on trials with correct responses and RTs $<1500 \mathrm{~ms}$. Explorative data analysis identified two participants that exhibited outlying task performance (ie, $d^{\prime}$ scores more than three standard deviations lower than the group mean) and were excluded from all analyses.

\section{Behavioral Results: Accuracy, RTs}

Behaviorally, participants discriminated between old and new items with high accuracy (Table 1) and without statistical differences between reward contingencies, as shown by a $2 \times 2$ ANOVA on $d^{\prime}$ scores, which revealed a main effect of group $(\mathrm{F}(1,34)=5.65, p=0.023)$ but no main effect of reward context $(\mathrm{F}(1,34)=0.84, p=0.366)$ and no interaction $(\mathrm{F}(1,34)=0.13, \quad p=0.719)$. The main effect of group was driven by increased $d^{\prime}$ scores (collapsed across contexts) in the placebo compared with the levodopa group $(t(1,34)=-2.38, p=0.023) \quad$ (see Table 1).

RT analysis revealed no main effect of novelty $(\mathrm{F}(1,34)=3.98, \quad p=0.054) \quad$ or group $(\mathrm{F}(1,34)=2.47$, $p=0.125)$, but a main effect of reward $(\mathrm{F}(1,34)=38.59$, $p=0.001)$ and an interaction of novelty $\times$ reward $(\mathrm{F}(1,34)=50.57, p=0.001)$. This interaction was due to faster RTs for reward predicting old images compared with not-reward predicting old images $(t(1,35)=-9.56$, $p=0.001)$. There were no significant RT differences for reward predicting new compared with not rewardpredicting new images $(t(1,35)=0.23, p=0.817)$. No other interactions reached statistical significance (all $p$ 's $>0.05$ ). See Table 1 for a complete list of $d^{\prime}$ scores and RTs.

There were no significant correlations between RTs and $d^{\prime}$ scores for the levodopa $(r=-0.361, p=0.141)$ or placebo group $(r=-0.202, p=0.421)$ arguing against a speed-accuracy trade-off in either group.

\section{MEG Results: Event-Related Fields}

We performed an initial $2 \times 2 \times 2$ ANOVA (group $\times$ novelty $\times$ reward, see Materials and methods) on the ERFs

Table I Behavioral Results

\begin{tabular}{|c|c|c|}
\hline & Levodopa M (SD) & Placebo M (SD) \\
\hline \multicolumn{3}{|l|}{$d^{\prime}$} \\
\hline CS + : old / & $1.65(0.75)$ & $1.95(0.83)$ \\
\hline \multicolumn{3}{|l|}{ CS - : new } \\
\hline CS + : new / & $1.76(0.75)$ & $2.19(0.58)$ \\
\hline \multicolumn{3}{|l|}{ CS - : old } \\
\hline \multicolumn{3}{|l|}{$R T$ (in $\mathrm{ms}$ ) } \\
\hline Old reward & $776.05(53.14)$ & $821.19(75.47)$ \\
\hline Old no reward & $871.86(62.31)$ & $903.59(68.63)$ \\
\hline New reward & $852.40(75.86)$ & $865.94(66.03)$ \\
\hline New no reward & 841.08 (63.27) & $872.80(80.05)$ \\
\hline
\end{tabular}

Abbreviations: $\mathrm{M}$, mean; SD, standard deviation.

Discrimination performance and reaction times. averaged across $60-140 \mathrm{~ms}$ to assess our prime hypothesis of an interaction between group and neural novelty processing at selected occipitotemporal sensors (see Materials and methods). This analysis revealed no main effects of group $(\mathrm{F}(1,34)=0.38, \quad p=0.541)$, novelty $(\mathrm{F}(1,34)=1.09, \quad p=0.304), \quad$ or reward $\quad(\mathrm{F}(1,34)=1.49$, $p=0.231)$ but, importantly, an interaction between group and novelty $(\mathrm{F}(1,34)=4.1, p=0.05)$. Post hoc analysis showed that this interaction was based on significantly stronger positive deflections for new compared with old stimuli in the placebo group $(t(1,17)=-2.17, p=0.044)$, but there were no novelty effects (ie, ERP differences to new $v s$ old items) in the levodopa group $(t(1,17)=0.69$, $p=0.497$ ) (Figure $2 b$ ). No other interaction reached statistical significance (all $p$ 's $>0.05$ ).

Visual inspection of the ERFs revealed a delayed onset of the novelty signal for the levodopa group centered at $\sim 650 \mathrm{~ms}$ (Figure $2 \mathrm{~b}$ ). To assess this effect statistically, another $2 \times 2 \times 2$ ANOVA (group $\times$ novelty $\times$ reward) on the ERFs (averaged across $500-800 \mathrm{~ms}$ ) was performed. It revealed significant main effects of group $(\mathrm{F}(1,34)=4.87$, $p=0.034)$, novelty $(\mathrm{F}(1,34)=9.14, p=0.005)$, and reward $(\mathrm{F}(1,34)=4.14, p=0.050)$, as well as an interaction between group and novelty $(\mathrm{F}(1,34)=5.54, p=0.025)$. This interaction was due to significantly stronger positive deflection for old in contrast to new items in the levodopa group $(t(1,17)=4.434, p<0.001)$, but there was no novelty effect in the placebo group $(t(1,17)=0.42, p=0.680)$ (Figure $2 \mathrm{~b}$ ). The main effect of reward was driven by stronger positive deflections for reward predicting $v s$ not-reward predicting stimuli $(t(1,35)=2.05, p=0.048)$ and is consistent with the fact that the preference judgment followed the initial old/new discrimination. No other interaction reached statistical significance (all $p$ 's $>0.05$ ).

Finally, we investigated the effects of drug on novelty and reward processing across all sensors and time-points $(-200-1000 \mathrm{~ms}$ ) by means of a $2 \times 2 \times 2$ ANOVA (group $\times$ novelty $\times$ reward, see Materials and methods) as implemented in SPM8. As we did not have any specific a priori hypotheses (outside the above-mentioned time-window), all statistical parametric maps were family-wise error (FWE) corrected $(p<0.05)$ to account for multiple statistical comparisons. This ANOVA revealed a main effect of group (F-contrast) over left occipital sensors peaking at $267 \mathrm{~ms}$ (Figure 3a; cluster size $k=55$ voxels; nearest sensor: MLO43; $p<0.05$ FWE-corrected). Closer inspection of this effect showed that it was driven by overall stronger positive deflections in the levodopa group in a time-window ranging from $\sim 100$ to $800 \mathrm{~ms}(t(1,34)=-3.026, p=0.005)$, irrespective of stimulus category (Figure $3 b$ ). No other main effects or interactions were observed before (ie., $-200-0 \mathrm{~ms}$ ) or after (ie, $0-1000 \mathrm{~ms})$ stimulus onset $(p<0.05$; FWEcorrected).

\section{DISCUSSION}

We investigated the role of dopamine in regulating the temporal dynamics of neural novelty processing. As hypothesized and in line with our previous findings (Bunzeck et al, 2009), in the placebo group we found significant old/new effects over temporal/occipital sensors 
peaking at $\sim 100 \mathrm{~ms}$ after stimulus onset. This remarkably early effect was not further accelerated by stimulating the dopaminergic system through levodopa but instead emerged delayed with an onset latency of $\sim 400 \mathrm{~ms}$ (Figure 2). Taken together with the fact that levodopa also impaired retrieval accuracy, this points towards a previously unreported role of dopamine in regulating the temporal dynamics and efficacy of neural processes that allow a distinction between old and new information.

Recognition memory studies in animals and humans have demonstrated divergent onset latencies for neural responses that distinguish new from old stimuli (ie, novelty signals). In non-human primates, intracranial recordings in the temporal lobe revealed novelty signals with an onset of $<100 \mathrm{~ms}$ (Brown and Xiang, 1998; Miller and Desimone, 1994), whereas in humans, earliest scalp-recorded novelty effects typically arise after $\sim 200 \mathrm{~ms}$ (Rugg and Curran, 2007). Although anatomical differences between species might contribute to this large temporal difference, it could recently be shown that another explanation lies in the necessity to utilize reward motivation in animals but not humans. More precisely, in animal studies the desired behavior to distinguish between new and old items is typically elicited by reinforcing either category using rewards, such as fruits or juice; in humans, on the other hand, verbal instructions are sufficient. In line with this notion, human scalp-recorded novelty responses can be accelerated from $\sim 200$ to $\sim 85 \mathrm{~ms}$ if the detection of either new or old items is rewarded (Bunzeck et al, 2009), which conforms to our current observation in the placebo group.

In the levodopa group, these novelty responses were not further accelerated but instead they were delayed to $\sim 400 \mathrm{~ms}$ after stimulus onset (peak at $\sim 650 \mathrm{~ms}$; Figure 2). This effect provides evidence for our hypothesis of a role of dopamine in the temporal control of neural old/new responses and can be reconciled on the basis of common neural substrates for reward and novelty processing within the dopaminergic mesolimbic system (Bunzeck and Düzel, 2006; Haber and Knutson, 2009; Lisman and Grace, 2005). In anatomical terms, the MTL, including the hippocampus, is assumed to generate a novelty signal if incoming information mismatches predictions. This novelty signal is sent to the SN/VTA, which releases dopamine back to the MTL where it drives synaptic plasticity and learning (Lisman and Grace, 2005; Lisman et al, 2011). On the other hand, the mesolimbic system is also well known for its role in reward processing whereby activity in the SN/VTA, MTL, and ventral striatum codes different aspects of reward learning, such as predictions and prediction errors (Haber and Knutson 2009; Holscher et al, 2003; Schultz et al, 1997). Taken together with the fact that contextual novelty modulates reward representations and vice versa (Bunzeck et al, 2012; Guitart-Masip et al, 2010), this clearly indicates the capability of the mesolimbic system to code and integrate different forms of motivationally relevant information.

A rather understudied form of plasticity that appears to be modulated by dopamine relates to the temporal properties of neural processing. We have recently shown that-in the absence of reward-neural novelty signals are accelerated to $<100 \mathrm{~ms}$ following levodopa administration (Eckart and Bunzeck, 2013). Under the assumption that reward prospect leads to dopamine release (Schott et al, 2008; Schultz et al, 1997; Tobler et al, 2005), these and our current results suggest that there is no linear but an inverted $\mathrm{u}$-shaped relationship between dopamine levels and the temporal dynamics of novelty processing (Figure 4 ). That means, with 'normal' dopamine levels (ie, in the absence of reward or pharmacological manipulations), old/new responses typically emerge at $\sim 200 \mathrm{~ms}$ after stimulus onset (Rugg and Curran, 2007); if dopamine levels are slightly enhanced to an 'optimum', either by pharmacological means (Eckart and Bunzeck, 2013) or in the context of reward motivation (Bunzeck et al, 2009), these old/new responses peak at $\sim 100 \mathrm{~ms}$. If, on the other hand, dopamine levels exceed the optimal range-as in our study, that is, in the context of reward together with levodopa administration-the onset of old/new responses is even slower than 'normal' and thus emerges not earlier than $\sim 400 \mathrm{~ms}$ after stimulus presentation. Importantly, this suggested inverted u-shaped relationship conforms to previous behavioral observation of faster old/new RTs in the prospect of reward (Bunzeck et al, 2009) and currently observed impaired retrieval accuracy (ie, lower $d^{\prime}$ values) when reward prospect is linked with levodopa administration.

Non-linear relationships between dopamine levels and cellular and/or cognitive functions are well known (eg, Goldman-Rakic et al, 2000; Cools and D'Esposito, 2011). For instance, in macaques the amount of receptor binding of prefrontal D1 receptors affects spatial tuning of neurons
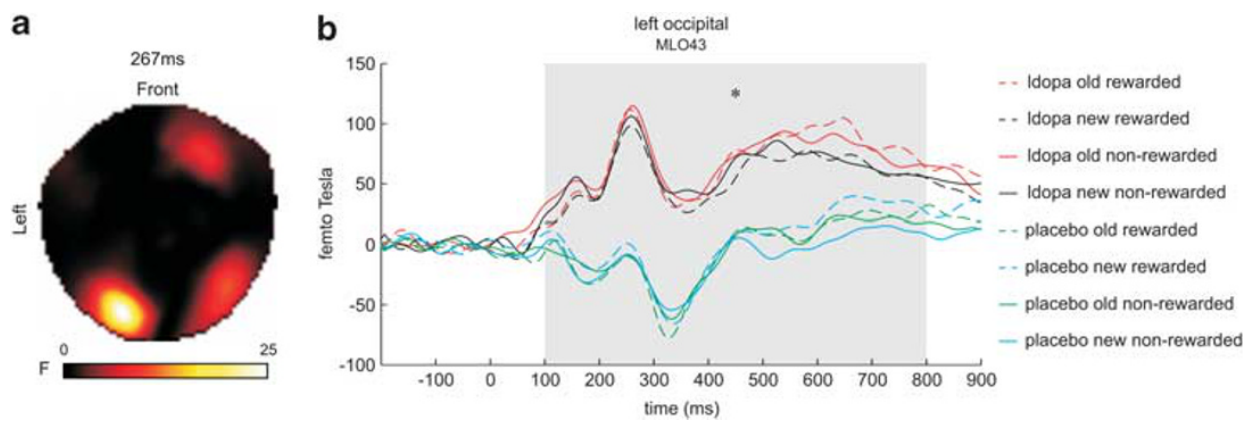

Figure 3 Main effect of drug group. Statistical Parametric Mapping-based analysis across all sensors and time-points revealed a main effect of drug group (levodopa, placebo) over left occipital sensors (peak at $267 \mathrm{~ms}$; nearest sensor: MLO43) (a). It was driven by more positive event-related magnetic fields across all conditions in the levodopa group ranging from $\sim 100$ to $800 \mathrm{~ms}$ (b). Asterisk indicates the statistically significant main effect $(p<0.05)$. 
engaged in working memory: while moderate levels enhance tuning, there is a reduction with low and high levels of D1 receptor occupancy (Williams and Goldman-Rakic, 1995). Largely motivated by these seminal findings, several psychopharmacological studies in humans have demonstrated that dopaminergic agonists can improve or worsen working memory performance (Kimberg et al, 1997; Mehta et al, 2000) and associated frontostriatal functional connectivity (Wallace et al, 2011), depending on individual baseline performance. Similarly, dose-dependent nonlinear effects of levodopa have been described for hippocampusdependent long-term memory in healthy older adults (Chowdhury et al, 2012). Therefore, our interpretation of an inverted u-shaped relationship between dopamine levels and the temporal dynamics of neural novelty processing (Figure 4) conforms to a wide range of previous animal and human research.

Although we do not have a direct measure of (drug-free) individual baseline performance, it seems unlikely that $d^{\prime}$ scores and onset latencies already differed between groups before drug administration since subjects were randomly assigned and data analyzed by using appropriate statistical measures. On a similar note, as there were no specific drug effects on reward signals, RTs or measures of subjective well-being, it appears unlikely that levodopa had general effects on neural processing or perceptual, cognitive or motor functions.

Previous work suggests that reward can enhance response vigor through dopaminergic mechanisms (Beierholm et al, 2013; Niv et al, 2007). Our behavioral data partly support this notion by demonstrating faster RTs to reward predicting $v s$ not-reward predicting old images. However, there was no such behavioral effect for new images and there was no modulation by levodopa. Although we do not take our data to argue against a role of reward in regulating response vigor, they clearly indicate a need to further explore the role of novelty and dopamine in this relationship (Kakade and Dayan, 2002).

The polarity for the two novelty effects reversed: in the early time-window (60-140 ms, placebo group), evoked responses to old stimuli were more positive compared with new stimuli, while during the later time-window (500-800 ms, levodopa group), the opposite pattern

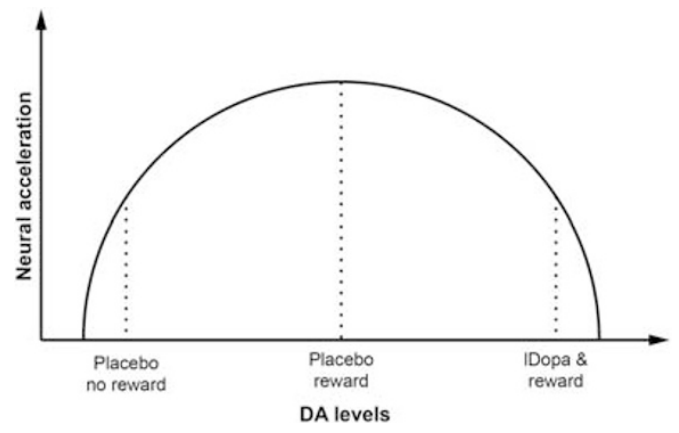

Figure 4 Inverted u-shaped relationship between dopamine (DA) levels and the chronometry of novelty processing. We suggest that slightly enhanced dopamine levels, for example, in the context of reward prospect (middle), accelerate the onset of neural novelty signals. If dopamine levels exceed an optimal range, for example, in the context of reward together with levodopa administration (right), the onset latency returns to baseline or even delays. emerged (Figure 2b). This observation is in accordance with previous work (Bunzeck et al, 2009; Eckart and Bunzeck, 2013) and suggests that diverging rather than the same neural population contributes to the generation of the early and later novelty signals. This and similar open questions, such as the relationship between the observed latency changes and hemodynamic effects, could be addressed more directly by combining functional magnetic resonance imaging (fMRI) with concurrent electroencephalography.

Although the sources of our scalp-recorded novelty signals can most likely be located to the MTL (Brown and Xiang, 1998; Eckart and Bunzeck, 2013; Miller and Desimone, 1994) rather than the dopaminergic midbrain, it is interesting to note that the temporal properties of the early effects are compatible with electrophysiological recordings in animals' SN/VTA. Specifically, midbrain dopamine neurons respond to novel (Ljungberg et al, 1992) or otherwise salient sensory stimuli (Horvitz et al, 1997) with a latency of $\sim 60-100 \mathrm{~ms}$ and endure for $<200 \mathrm{~ms}$. Similar response profiles have been shown for reward and reward predicting cues (Schultz, 2007).

The analysis across all sensors and time-points revealed a main effect of levodopa over left occipital sensors ranging from $\sim 100$ to $\sim 800 \mathrm{~ms}$ after stimulus onset (Figure 3). It was driven by stronger positive ERFs in the levodopa group independent of stimulus category and therefore might relate to overall enhanced stimulus salience (Berridge, 2007). Indeed, elevated dopamine levels can lead to aberrant signal processing, resulting in excessive assignment of saliency to external and internal stimuli (Kapur, 2003; Lodge and Grace, 2007). In line with this notion, a recent fMRI study in healthy humans could show that stimulation with $\triangle 9$-THC, a substance that is known to induce striatal dopamine release (Bossong et al, 2009), augmented cortical activation in response to salient stimuli (Bhattacharyya et al, 2012). Taken together with observations of increased neural signals in the MTL (Bunzeck et al, 2013) and prefrontal cortex (Eckart and Bunzeck, 2013) following levodopa administration, our current findings further indicate a close link between enhanced dopamine levels and stimulus salience.

To summarize, we can demonstrate that levodopa administration delays early neural novelty responses in the context of reward prospect and impairs the ability to discriminate between old and new information. This indicates a previously unknown causal role of dopamine in regulating the chronometry of neural memory signals and associated retrieval accuracy. We suggest that this relationship follows an inverted u-shaped function whereby only slightly enhanced dopamine levels accelerate neural and cognitive functions; excessive dopamine levels, on the other hand, impair them.

\section{FUNDING AND DISCLOSURE}

The authors declare no conflict of interest.

\section{ACKNOWLEDGEMENTS}

This work was supported by Hamburg state cluster of excellence (neurodapt!). We thank Vanessa Rausch for 
support in data acquisition and helpful comments on the manuscript.

\section{REFERENCES}

Beierholm U, Guitart-Masip M, Economides M, Chowdhury R, Duzel E, Dolan R et al (2013). Dopamine modulates reward related vigor. Neuropsychopharmacology 38: 1495-1503.

Berridge KC (2007). The debate over dopamine's role in reward: the case for incentive salience. Psychopharmacology (Berl) 191: 391-431.

Bhattacharyya S, Crippa JA, Allen P, Martin-Santos R, Borgwardt S, Fusar-Poli $\mathrm{P}$ et al (2012). Induction of psychosis by $\Delta$ 9-tetrahydrocannabinol reflects modulation of prefrontal and striatal function during attentional salience processing. Arch Gen Psychiatry 69: 27-36.

Bossong MG, Berckel BNM, van Boellaard R, Zuurman L, Schuit RC, Windhorst $\mathrm{AD}$ et al (2009). Delta 9-tetrahydrocannabinol induces dopamine release in the human striatum. Neuropsychopharmacology 34: 759-766.

Brown MW, Xiang JZ (1998). Recognition memory: neuronal substrates of the judgement of prior occurrence. Prog Neurobiol 55: $149-189$.

Bunzeck N, Doeller CF, Dolan RJ, Duzel E (2012). Contextual interaction between novelty and reward processing within the mesolimbic system. Hum Brain Mapp 33: 1309-1324.

Bunzeck N, Doeller CF, Fuentemilla L, Dolan RJ, Duzel E (2009). Reward motivation accelerates the onset of neural novelty signals in humans to 85 milliseconds. Curr Biol 19: 1294-1300.

Bunzeck N, Düzel E (2006). Absolute coding of stimulus novelty in the human substantia nigra/VTA. Neuron 51: 369-379.

Bunzeck N, Guitart-Masip M, Dolan RJ, Duzel E (2013). Pharmacological dissociation of novelty responses in the human brain. Cereb Cortex (doi:10.1093/cercor/bhs420).

Chowdhury R, Guitart-Masip M, Bunzeck N, Dolan RJ, Düzel E (2012). Dopamine modulates episodic memory persistence in old age. J Neurosci 32: 14193-14204.

Cools R, D'Esposito M (2011). Inverted-U-shaped dopamine actions on human working memory and cognitive control. Biol Psychiatry 69: e113-e125.

Devenport LD, Devenport JA, Holloway FA (1981). Rewardinduced stereotypy: modulation by the hippocampus. Science 212: 1288-1289.

Eckart C, Bunzeck N (2013). Dopamine modulates processing speed in the human mesolimbic system. NeuroImage 66: 293-300.

Goldman-Rakic P, Muly I, Williams G (2000). D1 receptors in prefrontal cells and circuits. Brain Res Rev 31: 295-301.

Guitart-Masip M, Bunzeck N, Stephan KE, Dolan RJ, Düzel E (2010). Contextual novelty changes reward representations in the striatum. J Neurosci 30: 1721-1726.

Guitart-Masip M, Chowdhury R, Sharot T, Dayan P, Duzel E, Dolan RJ (2012). Action controls dopaminergic enhancement of reward representations. Proc Natl Acad Sci USA 109: 7511-7516.

Haber SN, Knutson B (2009). The reward circuit: linking primate anatomy and human imaging. Neuropsychopharmacology 35: 4-26.

Holscher C, Jacob W, Mallot HA (2003). Reward modulates neuronal activity in the hippocampus of the rat. Behav Brain Res 142: 181-191.

Horvitz JC, Stewart T, Jacobs BL (1997). Burst activity of ventral tegmental dopamine neurons is elicited by sensory stimuli in the awake cat. Brain Res 759: 251-258.

Kakade S, Dayan P (2002). Dopamine: generalization and bonuses. Neural Networks 15: 549-559.
Kapur S (2003). Psychosis as a state of aberrant salience: a framework linking biology, phenomenology, and pharmacology in schizophrenia. Am J Psychiatry 160: 13-23.

Kimberg DY, D'Esposito M, Farah MJ (1997). Effects of bromocriptine on human subjects depend on working memory capacity. NeuroReport 8: 3581-3585.

Lisman J, Grace AA, Duzel E (2011). A neoHebbian framework for episodic memory; role of dopamine-dependent late LTP. Trends Neurosci 34: 536-547.

Lisman JE, Grace AA (2005). The hippocampal-VTA loop: controlling the entry of information into long-term memory. Neuron 46: 703-713.

Litvak V, Mattout J, Kiebel S, Phillips C, Henson R, Kilner J et al (2011). EEG and MEG data analysis in SPM8. Comput Intell Neurosci 2011: 852-961.

Ljungberg T, Apicella P, Schultz W (1992). Responses of monkey dopamine neurons during learning of behavioral reactions. J Neurophysiol 67: 145-163.

Lodge DJ, Grace AA (2007). Aberrant hippocampal activity underlies the dopamine dysregulation in an animal model of schizophrenia. J Neurosci 27: 11424-11430.

Mehta MA, Calloway P, Sahakian BJ (2000). Amelioration of specific working memory deficits by methylphenidate in a case of adult attention deficit/hyperactivity disorder. J Psychopharmacol 14: 299-302.

Miller EK, Desimone R (1994). Parallel neuronal mechanisms for short-term memory. Science 263: 520-522.

Niv Y, Daw ND, Joel D, Dayan P (2007). Tonic dopamine: opportunity costs and the control of response vigor. Psychopharmacology (Berl) 191: 507-520.

Nyholm D, Lewander T, Gomes-Trolin C, Bäckström T, Panagiotidis G, Ehrnebo M et al (2012). Pharmacokinetics of levodopa/ carbidopa microtablets versus levodopa/benserazide and levodopa/carbidopa in healthy volunteers. Clin Neuropharmacol 35: 111-117.

O’Doherty JP (2004). Reward representations and reward-related learning in the human brain: insights from neuroimaging. Curr Opin Neurobiol 14: 769-776.

Rolls ET, Xiang J-Z (2005). Reward-spatial view representations and learning in the primate hippocampus. J Neurosci 25: 6167-6174.

Rugg MD, Curran T (2007). Event-related potentials and recognition memory. Trends Cogn Sci (Regul Ed) 11: 251-257.

Schott BH, Minuzzi L, Krebs RM, Elmenhorst D, Lang M, Winz OH et al (2008). Mesolimbic functional magnetic resonance imaging activations during reward anticipation correlate with reward-related ventral striatal dopamine release. J Neurosci 28: 14311-14319.

Schultz W (2007). Multiple dopamine functions at different time courses. Annu Rev Neurosci 30: 259-288.

Schultz W, Dayan P, Montague PR (1997). A neural substrate of prediction and reward. Science 275: 1593-1599.

Stanislaw H, Todorov N (1999). Calculation of signal detection theory measures. Behav Res Methods Instrum Comput 31: 137-149.

Strange BA, Fletcher PC, Henson RNA, Friston KJ, Dolan RJ (1999). Segregating the functions of human hippocampus. Proc Natl Acad Sci USA 96: 4034-4039.

Tobler PN, Fiorillo CD, Schultz W (2005). Adaptive coding of reward value by dopamine neurons. Science 307: 1642-1645.

Wallace DL, Vytlacil JJ, Nomura EM, Gibbs SEB, D’Esposito M (2011). The dopamine agonist bromocriptine differentially affects fronto-striatal functional connectivity during working memory. Front Hum Neurosci 5: 32.

Williams GV, Goldman-Rakic PS (1995). Modulation of memory fields by dopamine D1 receptors in prefrontal cortex. Nature 376: $572-575$. 
Wimmer GE, Shohamy D (2012). Preference by association: how memory mechanisms in the hippocampus bias decisions. Science 338: $270-273$.

Wittmann BC, Bunzeck N, Dolan RJ, Duzel E (2007). Anticipation of novelty recruits reward system and hippocampus while promoting recollection. NeuroImage 38: 194-202.
Worsley KJ, Taylor JE, Tomaiuolo F, Lerch J (2004). Unified univariate and multivariate random field theory. Neuroimage 23(Suppl 1): S189-S195.

Yamaguchi S, Hale LA, D’Esposito M, Knight RT (2004). Rapid prefrontal-hippocampal habituation to novel events. J Neurosci 24: 5356-5363.

Supplementary Information accompanies the paper on the Neuropsychopharmacology website (http://www.nature.com/npp) 